\title{
Wisconsin In-Situ Penning (WISP) Gauge A versatile neutral pressure gauge to measure partial pressures in strong magnetic fields
}

\author{
T. Kremeyer, ${ }^{1,}$ a) K. Flesch, ${ }^{1}$ O. Schmitz, ${ }^{1}$ G. Schlisio, ${ }^{2}$ U. Wenzel, ${ }^{2}$ and W7-X Team ${ }^{2, b)}$ \\ 1) University of Wisconsin - Madison, Madison, WI 53706, USA \\ 2) Max Planck Inst. for Plasma Physics, 17491 Greifswald, Germany
}

(Dated: 14 April 2020)

A new type of in-vessel Penning gauge, the Wisconsin In-Situ Penning (WISP) gauge, has been developed and successfully operated in the Wendelstein 7-X (W7-X) island divertor baffle and vacuum vessel. The capacity of the quantitative measurements of the neutral reservoir for light impurities, in particular helium, is important for tokamaks as well as stellarator divertors in order to avoid fuel dilution and radiative energy loss. Penning gauges assisted by spectroscopy are a powerful tool to obtain the total neutral pressure as well as fractional neutral pressures of specific impurities. The WISP gauge is a miniaturized Penning gauge arrangement, which exploits the ambient magnetic field of magnetic confinement fusion experiments to establish the Penning discharge. Then, in-situ spectroscopy is conducted to separate the fractional neutral pressures of hydrogen, helium and possibly also other impurities. The WISP probe head was qualified using the magnetic field of the Magnetized Dusty Plasma Experiment, MDPX, at Auburn University between 0.25 T and 3.5 T [E. Thomas, Journal of Plasma Physics 81 (2015)]. The in-depth quantitative evaluation for hydrogen and helium will be shown as well as an exploration of nitrogen, argon and neon. A power law scaling between current $I$ and pressure $p: I=f(\mathrm{Gas}, \mathrm{V}) \cdot p^{n(\mathrm{Gas}, \mathrm{B})}$ was shown. The factor $f$ is gas and anode potential dependent, while $n$ is gas and magnetic field strength dependent. Pressure measurements from 0.1 mbar and down to $1 \times 10^{-5}$ mbar were achieved, demonstrating a reliable operation range for relevant pressure levels in the divertor and main vessel regions in current and future fusion devices, with a time resolution of up to $1 \mathrm{kHz}$. The lowest achievable pressure measurement increases with increasing $B$ and can be shifted with the anode potential $V$.

At Wendelstein 7-X the WISP probe head was mounted on an immersion tube set up that reaches through the cryostat and places the probe head close to the plasma. Two probe heads were positioned in the different divertor pump gaps, top and bottom, and one close to the plasma on the mid-plane in one module. The gauges were in-situ calibrated together with the ASDEX pressure gauges [G. Haas and H. Bosch, Vacuum 51, 39 (1998)]. Data was taken during the entire operation phase 1.2b (OP1.2b) and measurements were coherent with other neutral gas pressure gauges. For the spectroscopic partial pressure measurements, channels of a spectroscopic detection system based on photo-multipliers, so-called Filterscope [R. Colchin, Review of scientific instruments 74, 2068 (2003)] provided by Oak Ridge National Lab (ORNL) were used.

\section{INTRODUCTION}

The fusion of deuterium (D) and tritium (T) in a burning fusion plasma will continuously produce alpha particles, leading to helium $(\mathrm{He})$ ash. The burn condition for D-T plasmas, limits the maximum He concentration to about $10 \%$, making a sufficient He exhaust mandatory for a reactor ${ }^{1}$. He exhaust can be divided into different regions, with each region requiring different diagnostics to measure the He concentration in it. The $\alpha$ particles are created in the plasma core from where they have to be transported into the edge and then outside the last closed flux surface (LCFS), neutralized at a divertor, pumped through the pumping gap, and eventually removed from the device through a pump duct. Charge exchange spectroscopy can be used to measure the He concentration in the plasma core ${ }^{2}$, edge, and $\mathrm{LCFS}^{3}$. Outside of

\footnotetext{
a) Author to whom correspondence should be addressed.kremeyer@wisc.edu; https://3dpsi.engr.wisc.edu/

${ }^{b)}$ For the complete member list, please refer to T. Klinger, et al., Nuclear Fusion, Volume 59, Number 11, 112004 (2019).
}

the machine in the pump duct several diagnostics have been developed to measure He partial pressures in a $\mathrm{D}_{2}$ environment. Even though many residual gas analyzers (RGA) that function on the principle of quadrupole mass spectrometers (QMS) are struggling to resolve the mass difference between $\mathrm{He}$ at $4.003 \mathrm{amu}$ and $\mathrm{D}_{2}$ at $4.028 \mathrm{amu}$, there are some commercial RGAs that claim to be capable of doing so. However they are sensitive to ambient magnetic field and thus might have to be mounted further away. Another technique is to excite the neutral gas while observing the discharge spectroscopically and using the line intensities for partial pressure measurements. Optical observations have been used on ASDEXtype hot cathode ion gauges ${ }^{4}$, while a comparison of different electron sources to excite the neutral gas has found that the best line emitting light sources is a Penning discharge ${ }^{5}$. Penning discharges 6 in combination with a spectroscopic optical observation have been used successfully at TEXTOR ${ }^{7}$,DIII$\mathrm{D}^{8}$, ASDEX-U, and $\mathrm{JET}^{9}$. However all these techniques have to be mounted on the outer periphery of the device to avoid interference with the devices magnetic field. Due to this, these techniques commonly struggle with having a response time compatible with plasma particle and impurity lifetimes in the divertor region. However clever vacuum engineering can mit- 

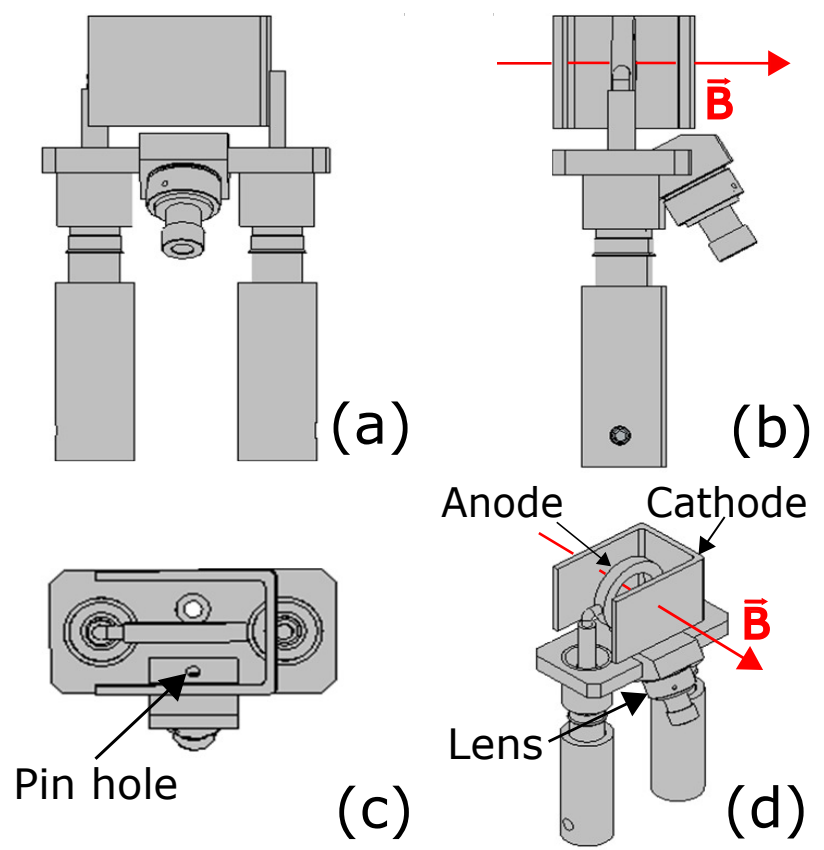

FIG. 1. CAD model of WISP Probe Head showing (a) front, (b) side, (c) top, and (d) isometric view

igate this effect ${ }^{10}$. The Wisconsin In-Situ Penning gauge was developed to fill this gap and offers a first in-situ partial neutral pressure measurement in magnetic fusion devices.

The paper has two main sections, in the first the WISP probe head itself as well as an in depth study of its behavior in different operating regimes is described. The second section describes how the probe head was implemented at W7-X and how it compares to ASDEX-type hot cathode ion gauges.

\section{THE WISP PROBE HEAD}

A previous anode design study described in reference 11 for in-situ Penning gauges was extended to develop the final probe head.The ring anode design was chosen over other geometries due to its superior performance in terms of stability and photon yield. The WISP probe head consists of a ring anode surrounded by a cathode plate. The anode and cathode are mechanically connected by a base plate where a lens is mounted and protected by a pin hole, as seen in the CAD model of the probe head in Fig 1 and Fig 2.The anode ring has an outer diameter of $9 \mathrm{~mm}$ and a $3 \mathrm{~mm}$ by $3 \mathrm{~mm}$ cross section. The cathode plate is made out of a bent plate with a thickness of $1.5 \mathrm{~mm}$. The anode, cathode, and base plate are all made out of stainless steel. The electrical insulation is achieved by custom boron nitride pieces and ceramic beads. The cathode plates are $17 \mathrm{~mm}$ apart from each other with the ring anode mounted in the center. The anode and cathode are supported from the side to keep the bottom view clear for the optical observation. To create a well-defined optical observation area, a combination of a pin hole camera with a collimator lens is used. The pin hole camera serves two functions. The main purpose is to protect the lens from deposition, which is achieved by making the hole as deep as its $2 \mathrm{~mm}$ diameter. The lens was then moved further back to increase the screening effect. The second function is that the combination of the

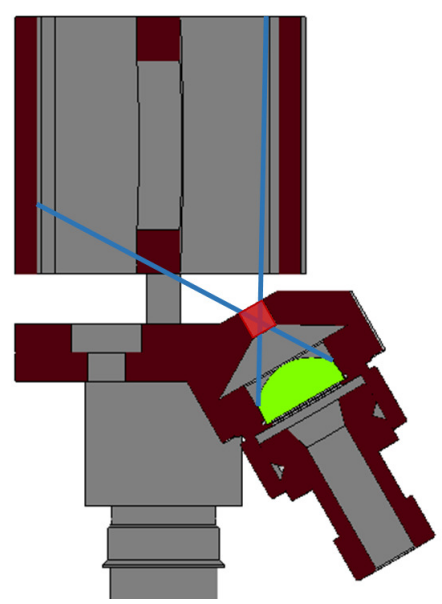

FIG. 2. Picture of a cross section of the WISP probe head with the lens in green, pin hole section in red and the field of view lines in blue

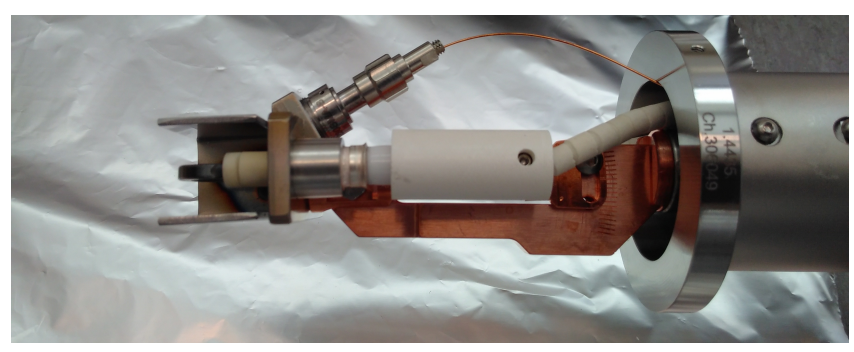

FIG. 3. Picture of a WISP probe head on top of an adjustment unit to align it to the magnetic field vector

pinhole together with the lens enables a well-defined field of view. The lens was positioned in a way that the lines of sight go through the anode ring and the majority of the discharge can be observed.

Commercial Penning gauges use permanent magnets to create their magnetic field for electron trapping. This can lead to interference when the gauge is operated close to the field of magnetic confinement machines. The WISP probe head avoids this issue by utilizing the present magnetic field of the device, and therefore can be placed directly inside the machine as long as the magnetic field vector is co-axial to the anode ring. A potential misalignment due to tolerances in the assembly or changes in the magnetic field are discussed in subsection III E. A picture of the probe head on an adjustment unit to align it in the magnetic field is shown in figure Fig. 3 For easier installation all connections were moved to the bottom of the base plate. In operation, high voltage is applied to the anode to create the electric field. For noise reduction the cathode plate is also insulated from the base plate,therefore also from machine ground, and has its own return. An optical fiber is connected to the collimator lens via an SMA connector and transmits the light to the spectroscopic device of choice.

The gauge has been designed and tested to resist the harsh environment of fusion devices. All materials and components are capable to be used under high vacuum conditions without outgasing. The lens, as the component with the lowest maximum temperature rating, is rated for temperatures of up 
to $180^{\circ} \mathrm{C}$ and thus allows the gauge to be baked at the typical temperatures for vacuum conditioning of about $150^{\circ} \mathrm{C}$. The metals used, have a magnetic permeability of $\mu_{r}<1.01$ to not perturb the local magnetic field and thus the measurement, and to avoid damaging the gauge due to a net force in the field. The cobalt concentration was kept below $0.05 \%$ to minimize activation in a neutron environment.

\section{WISP BEHAVIOR IN DIFFERENT SCENARIOS}

The WISP probe head went through an in depth testing at the Magnetized Dusty Plasma Experiments (MDPX) at Auburn University ${ }^{12}$. The superconducting MDPX magnet offers the unique capability of a freely adjustable magnetic field strength between 0 and $3.5 \mathrm{~T}$. The vacuum chamber was replaced for the systematic testing described in this paper with an ultra high vacuum chamber based on a 6-way ConFlat cross and ConFlat components. For pressure measurements and vacuum monitoring an Agilent FRG-700 Pirani Inverted Magnetron gauge together with an RGA were mounted, away from the field, close to the pump. The actual chamber was equipped with a MKS 722 B baratron $\left(1 \times 10^{3}\right.$ mbar to $\left.1 \times 10^{-4} \mathrm{mbar}\right)$ and an KJLC 354 ion gauge $\left(6.7 \times 10^{-2} \mathrm{mbar}\right.$ to $\left.1.3 \times 10^{-9} \mathrm{mbar}\right)$ measuring a base pressure of $2.6 \times 10^{-6}$ mbar. All following pressure measurements were performed with the baratron since the ion gauge could not be operated in magnetic fields and was only used for vacuum verification before the field was ramped up. The baratron was positioned in close vicinity of the WISP probe head so that dynamic effects can be neglected. A He and a $\mathrm{H}$ gas feed were connected to the chamber, that were controlled using mass flow controllers (MFC). Two high voltage power supplies were used to apply the high voltage to the anode and to measure the ion current. Most measurements were taken with a $0-5 \mathrm{kV}$ at a maximum of $60 \mathrm{~mA}$ power supply while a $0-2 \mathrm{kV}$ at a maximum of $150 \mathrm{~mA}$ power supply was used to determine the maximum measurable pressure. The spectroscopy was done using a USB CCD spectrometer with an integration time of $600 \mathrm{~ms}$ and monitored the $H_{\alpha}$ line and four different He-I lines at $587 \mathrm{~nm}, 668 \mathrm{~nm}, 706 \mathrm{~nm}$, and $728 \mathrm{~nm}$, based on previous studies ${ }^{7}$. The WISP probe head was tested at 8 different field strengths (Subsection III A), at 7 different anode potentials (Subsection III B), and in H and He (Subsection IIIC). Different mixtures of $\mathrm{H}$ and $\mathrm{He}$ were tested (Subsection III D) as well as a systematic misalignment of the probe head to the field to determine mounting tolerances as well as effects of changes in the magnetic field direction (Subsection IIIE). Due to the large amount of data taken, only a few exemplary sets will be discussed in each section. At W7$\mathrm{X}$ a calibration was done at the beginning and at the end of the campaign and the aging effects are discussed in subsection III F.

The ion current $I$ behavior, which is the measurement proportional to the neutral pressure $p$, can be approximated by a power law scaling.

$$
I=f(\mathrm{Gas}, \mathrm{V}) \cdot p^{n(\mathrm{Gas}, \mathrm{B})}
$$

However, at the lower end of the operating regime, the currents are lower in $\mathrm{H}$, and higher in $\mathrm{He}$, than predicted by the scaling. The line intensities are proportional to the ion

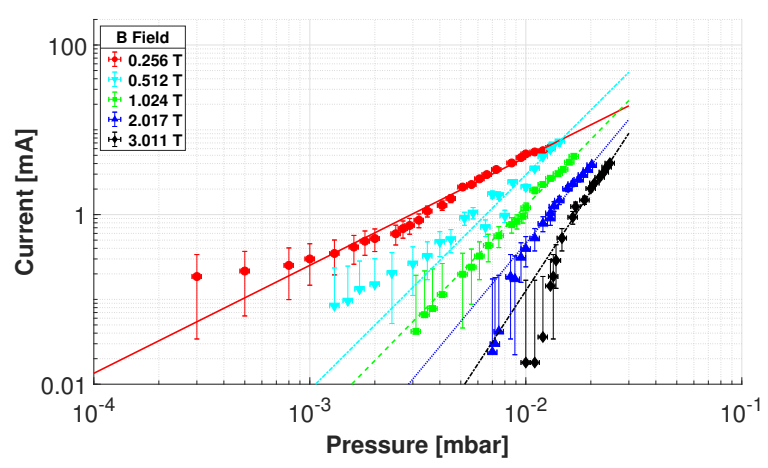

FIG. 4. Measured ion current for pressure sweeps in $\mathrm{He}$ at various B field strengths with anode at $1500 \mathrm{~V}$

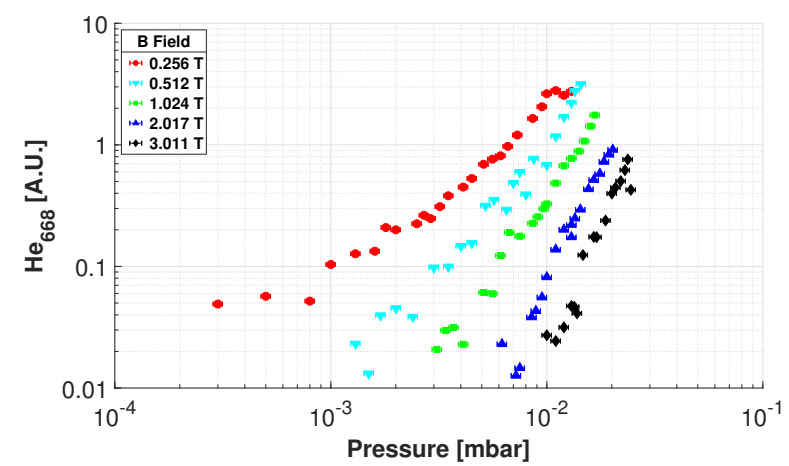

FIG. 5. B field comparison of He $668 \mathrm{~nm}$ line intensity measurements over pressure of WISP probe head in $\mathrm{He}$ at $1500 \mathrm{~V}$ with an integration time $T_{\text {int }}=600 \mathrm{~ms}$

current. However this systematic deviation was only seen at MDPX and neither at the PAX magnet ${ }^{13}$, nor at the calibration at W7-X, where the measurements were true to the scaling even at the lowest measureable pressures. It can therefor be concluded that this deviation is unique to the experimental set up at MDPX. Because of this, the plots in this paper show the general behavior of the probe head in different scenarios but can not serve as a calibration function. An in-situ calibration in the measuring position at the particular device is inevitable.

\section{A. Magnetic field scan}

Current (Fig. 4) as well as line intensity (Fig. 5) measurements over neutral pressure were taken at eight different magnetic field strengths, starting at $0.25 \mathrm{~T}, 0.5 \mathrm{~T}$ and then going up in half Tesla steps, up to $3.5 \mathrm{~T}$. Each curve represents a different magnetic field strength going from weaker to stronger from left to right. The power law scaling from equation (1) fits well with all adjusted $\mathrm{R}$-square values above 0.95 with the exception of the $0.512 \mathrm{~T}$ case which has an adjusted R-squared value of 0.78 and is likely due to a systematic error in the gas inlet/pressure measurement of that data set. At low pressures the measured current is higher for $\mathrm{He}$ and lower for $\mathrm{H}$, than predicted by the fit as mentioned in section III. This behavior is most likely due to the vacuum and gas feed system at MDPX since this behavior was not seen at other vacuum test stands or at $\mathrm{W} 7-\mathrm{X}$. The error in current increases relative to 


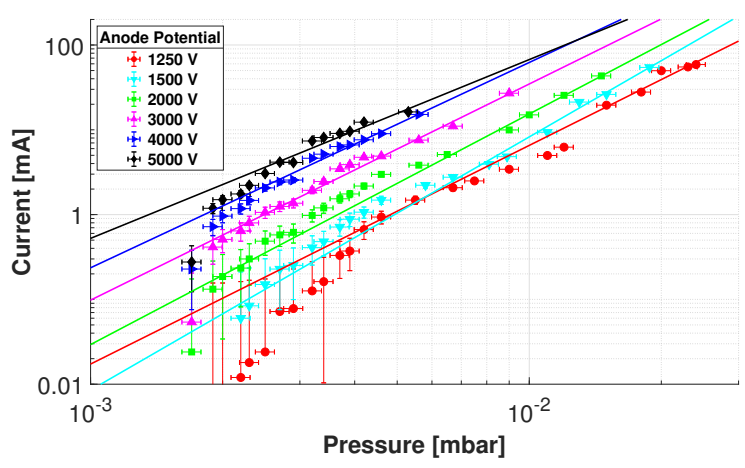

FIG. 6. Ion current over pressure of WISP probe head in $\mathrm{H}$ at 1.024 $\mathrm{T}$ at different potentials

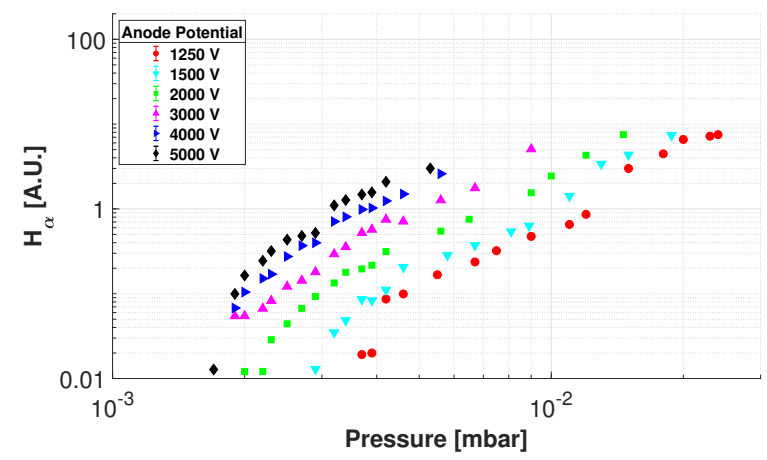

FIG. 7. $H_{\alpha}$ intensity over pressure of WISP probe head in $\mathrm{H}$ at 1.024 $\mathrm{T}$ at different potentials

the measured current at lower currents due to a systematic error of the ammeter which is constant throughout its operating range. A more sensitive ammeter could be used to increase accuracy at lower pressures. With increasing field the lower end of the operating regime shrinks. The $I(p)$ curves are getting steeper while reaching comparable upper pressures. The data in Fig. 5 is from the same settings as that in Fig. 4, but displays the $668 \mathrm{~nm} \mathrm{He}-\mathrm{I}$ line instead of ion current. The line intensity is proportional to the ion current shown in Fig 4 and follows the same characteristics.

The exponent $n(G a s, B)$ of the power law scaling in equation (1) can be approximated as equation (2) for $\mathrm{H}$ and equation (3) for $\mathrm{He}$ based on fit parameters. The adjusted $R^{2}$ values are 0.88 for $\mathrm{H}$ (Eq. 2) and 0.86 (Eq. 3) for He.

$$
\begin{aligned}
& n=1.9 \cdot B[\mathrm{~T}]+0.85 \\
& n=0.8 \cdot B[\mathrm{~T}]+1.40
\end{aligned}
$$

\section{B. Anode potential scan}

The WISP probe head was run at 7 different anode potentials: $1.25 \mathrm{kV}, 1.5 \mathrm{kV}, 1.75 \mathrm{kV}, 2 \mathrm{kV}, 3 \mathrm{kV}, 4 \mathrm{kV}$, and $5 \mathrm{kV}$, as seen in Fig. 6 and Fig. 7 . Fig. 6 shows the ion current and Fig. 7 the $H_{\alpha}$ line intensity plotted over the neutral pressure in $\mathrm{H}$ at a constant field of $1.024 \mathrm{~T}$. The pressure sweeps were conducted for each of the 7 anode potentials. The pressure curves can be approximated by the power law scaling (1)

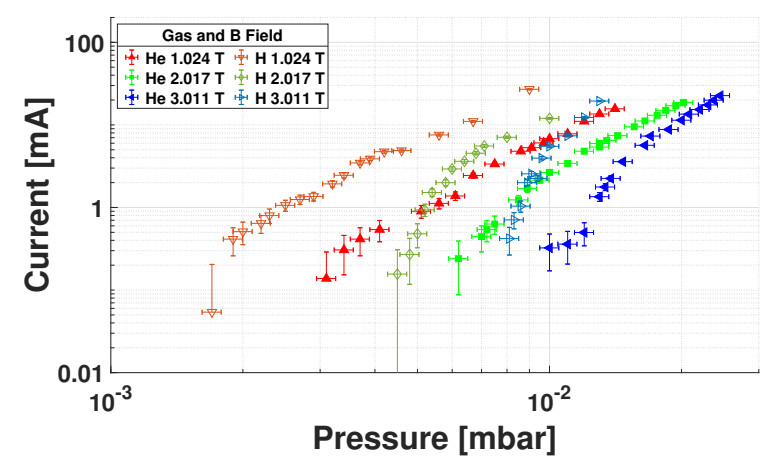

FIG. 8. Comparison of $\mathrm{H}$ and $\mathrm{He}$ ion current measurements over pressure of WISP probe head at $3000 \mathrm{~V}$

starting with the curve of the lowest potential on the right, going to the one with the highest potential on the left. The adjusted R-square values are above 0.95 for all fits, however it should be noted that at the lower end of the operating regime the power law scaling (1) overestimates the ion current. This effect is only seen in $\mathrm{H}$, the cause for this is still under investigation. The anode potential $V$ can be used as an easy tool to shift the operating regime. A higher potential will increase the ion current, ignite a discharge at lower pressures and therefore shift the measurement range to the left.

The ion current scales linearly as shown in equation (4).

$$
f(V)=3.6 \cdot V[\mathrm{kV}]
$$

For high pressures a lower potential is desirable to minimize the power that is being deposited in the probe head and prevent overheating. A lower potential will generate a lower ion current at the same pressure as a higher potential. A lower potential directly lowers the power and also lowers the ion current at a given pressure and therefore decreases the input power $P=V \cdot I$ significantly. An overheating of the probe head can lead to a glow of the anode which can interfere with the spectroscopic measurements and eventually melt and therefore destroy the anode.

\section{Gas dependence of ion current measurement}

Like any ion gauge, the WISP gauges ion current measurement is gas dependent. At the same neutral pressure nitrogen (N) results in a larger ion current than $\mathrm{H}$ which is larger than $\mathrm{He}$. Figure 8 shows a comparison of $\mathrm{H}$ and $\mathrm{He}$ measurements at different magnetic field strengths. While commercial Penning gauges are usually calibrated to Nitrogen and use a calibration factor for different gases, such a factor could not be identified for the WISP gauge. One reason for this is the gas dependence of the exponent as shown in equation (2) and (3). For a certain magnetic field strength and anode potential an approximation can be made where, depending on the case, the ion current scales between $I_{\mathrm{H}}=4 \cdot I_{\mathrm{He}}$ and $I_{\mathrm{H}}=8 \cdot I_{\mathrm{He}}$, however no general function between the gas species and the factor $f(\mathrm{Gas}, \mathrm{V})$ from equation (1) could be found.

While an ion gauge can use a calibration factor, or like in the case of the WISP gauge, can be calibrated for each gas species, all ion gauges struggle with accurate measurements in 
TABLE I. Observed atomic spectral lines in the visible range inside the WISP head in $\mathrm{nm}$. Lines are listed from strongest to weakest.

\begin{tabular}{ccccc}
\hline \hline $\mathrm{H}$ & $\mathrm{He}$ & $\mathrm{Ne}$ & $\mathrm{N}$ & $\mathrm{Ar}$ \\
\hline 656.1 & 587.6 & 586.8 & 391.9 & 812.8 \\
& 667.8 & 640.2 & 469.5 & 751.6 \\
& 706.5 & 614.3 & 869.3 & 764.7 \\
& 728.1 & 650.7 & 524.9 & 773.4 \\
\hline \hline
\end{tabular}

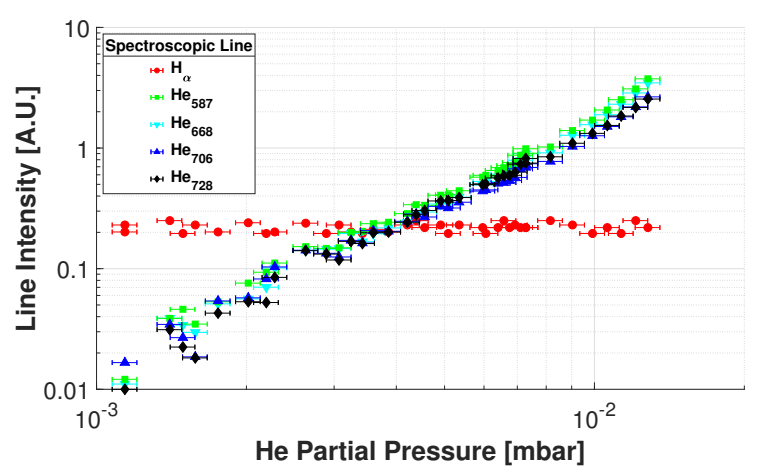

FIG. 9. $H_{\alpha}$ and He-I line intensities at $T_{\text {int }}=600 \mathrm{~ms}$ over pressure of WISP probe head at $2 \mathrm{kV}, 1.024 \mathrm{~T}$ and $3 \times 10^{-3}$ mbar of $\mathrm{H}$

gas mixtures. While the effect is negligible for trace amounts it becomes more severe with an increasing fraction of the other gases. The WISP gauge can avoid this problem by using the spectroscopic signal as a partial pressure measurement as described in subsection III D.

\section{Partial pressure scan}

For exhaust measurements, H/D and He partial pressure measurements are the most important but Ne, N, and Ar measurements can be important since they are used for diagnostic use $^{14}$ and edge cooling ${ }^{15}$. Possible lines for partial pressure measurements, which were actually observed in the gauge, are listed in Table I.

As described in subsection III C, the gas dependence of the ion current measurement increases the error of an ion gauge measurement in gas mixtures. This problem can be avoided with the WISP gauge or other optical Penning gauges, by extracting partial pressures from ratios of spectral line intensities after calibration with known gas mixtures.

As long as all present species are accounted for, adding all the partial pressures together will result in an accurate total neutral pressure measurement independent of gas mixture.

Figure 9

shows a pressure scan of He with a constant background pressure of $\mathrm{H}$ to study the cross-talk between the two gas species. Due to limitations in the vacuum and $\mathrm{H}$ supply system only a limited flow rate of $\mathrm{H}$ was available. This lead to $\mathrm{H}$ becoming the minority species as the He partial pressure was continuously increased. While it is less common to operate optical Penning gauges with $\mathrm{H}$ as the minority species, it has been used to study hydrogen coming off the wall during He wall conditioning at $\mathrm{TCV}^{16}$. This could lead to a different operation regime, since the ionization of $\mathrm{He}$ is providing the

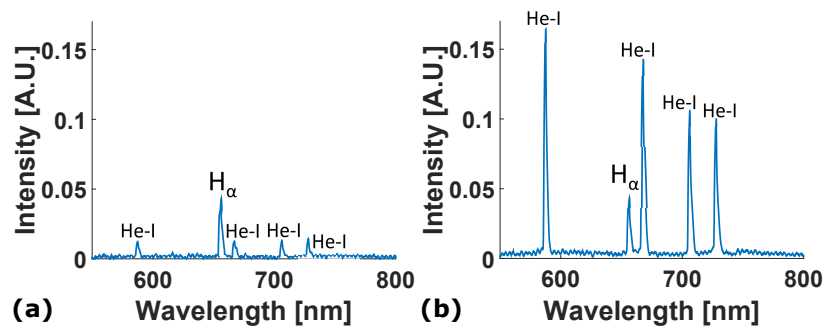

FIG. 10. Spectra taken at $T_{i n t}=600 \mathrm{~ms}$ with WISP probe head at $2 \mathrm{kV}, 1.024 \mathrm{~T}$ and a constant $\mathrm{H}$ partial pressure of $3 \times 10^{-3} \mathrm{mbar}$. (a) is taken with a He partial pressure of $2 \times 10^{-3}$ mbar and (b) with $1 \times 10^{-2} \mathrm{mbar}$

electrons to sustain the discharge.

$$
\begin{gathered}
\left(\begin{array}{c}
p_{\mathrm{H}} \\
p_{\text {Impurity }}
\end{array}\right)=A \cdot\left(\begin{array}{c}
I_{H \alpha} \\
I_{\text {Impurity }}
\end{array}\right) \\
A=\left(\begin{array}{cc}
\text { Linear H response } & \text { Impurity cross talk into H line } \\
\mathrm{H} \text { cross talk into impurity line } & \text { Linear impurity response }
\end{array}\right)
\end{gathered}
$$

However, no mode switching or change in in operation regime was found at W7-X where measurements of He as a minority and majority species were achieved. A scan going from $0 \%$ $\mathrm{He}$ to $50 \% \mathrm{He}$ in a $\mathrm{H}$ environment is discussed later in figure 21. In figure 9, four He-I lines and the $H_{\alpha}$ line are plotted over the He partial pressure. All He-I line intensities increase with pressure while the $H_{\alpha}$ line intensity stays constant within normal variations.

For partial pressure measurements the lines have to be chosen carefully to avoid contamination through neighboring lines, like the $586.8 \mathrm{~nm} \mathrm{Ne}$ line and the $587.6 \mathrm{~nm} \mathrm{He}$ line.
There can also be cross-talk from molecular hydrogen at this wavelength but the amount at the $667.8 \mathrm{~nm}$ line is negligible ${ }^{7}$. All listed He I lines show a very similar line intensity as seen in Fig. 9, so any other line is preferred. Figure 9 and figure 10 also show that the $H_{\alpha}$ signal is not affected by the He. The $\mathrm{H}_{2}$ pressure is linear to the intensity of the $H_{\alpha}$ line directly determined from the measured spectra, as seen in Fig.6 and Fig.7. If the cross talk cannot be avoided by the selection of lines, it will have to be accounted for with a calibration matrix (Eq. 6) as shown in equation (5).

Spectra of two data points from the He pressure scan with a 


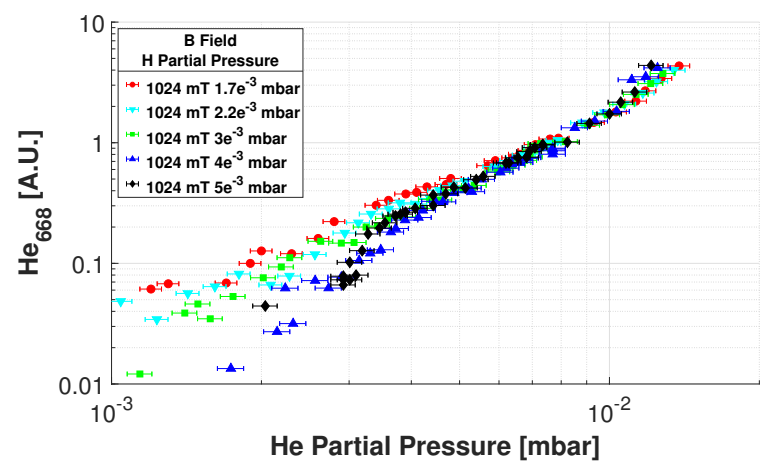

FIG. 11. $\mathrm{He}_{668}$ line intensity at $T_{i n t}=600 \mathrm{~ms}$ over pressure of WISP probe head at $2 \mathrm{kV}$ and different partial pressures of $\mathrm{H}$

constant $\mathrm{H}$ background pressure of $3 \times 10^{-3}$ mbar are shown in figure 10. Spectrum (a) on the left is taken with a He partial pressure of $2 \times 10^{-3} \mathrm{mbar}$ and was increased to $1 \times 10^{-2} \mathrm{mbar}$ for spectrum (b). The $H_{\alpha}$ line and the four He-I lines from table I are labeled. The increase of the He-I line intensities due to the increase in He partial pressure shows no significant effect on the $H_{\alpha}$ intensity, which stays constant. The spectra also show the absence of disturbing lines and further support that the crosstalk between $\mathrm{H}$ and $\mathrm{He}$ is negligible.

To empirically test for cross talk between $\mathrm{H}$ and $\mathrm{He}, \mathrm{He}$ pressure scans were done with different constant $\mathrm{H}$ background pressures. Figure 11

shows that the $\mathrm{H}$ cross talk into any of the He lines is negligible. Cross talk might be present at lower pressures as Fig. 11 could indicate that the lower He partial pressures are only accessible for lower $\mathrm{H}$ content. However this is counter intuitive as a lower $\mathrm{H}$ partial pressure also means a lower total pressure and therefore a reduction of the overall ion current and a weaker discharge. Future work and modeling will focus on assessing this behavior at lower pressures. Based on this any line intensity is proportional to the partial pressure of $\mathrm{He}$ in the majority of the operating range and can be used for partial pressure measurements, as long as there are no other species with neighboring lines present. Care must be taken at the lower end of the operating regime, until the cross talk behavior is completely understood or it has to be accounted for with Eq. 5 and Eq. 6.

Optical studies on commercial Penning gauges in hydrogen observed a saturation in the $D_{\alpha}$ signal and it was also shown to be different for the atomic and for the molecular contributions to the excitation of the Balmer- $\alpha$ lines ${ }^{17}$. The WISP probe head operated in a He majority shows no saturation up to $1 \times 10^{-2} \mathrm{mbar}$, as seen in figure 9 and 11 . Future work will try to investigate if this is due to the different geometry, the optimization of the Penning operational parameters, or the atomic physics.

\section{E. Misalignment test}

In magnetic confinement machines, the magnetic field topology can change during discharges due to a number of reasons, including bootstrap currents and running a different field configuration. This can lead the magnetic field vector to not be co-axially aligned to the WISP probe head anymore. To

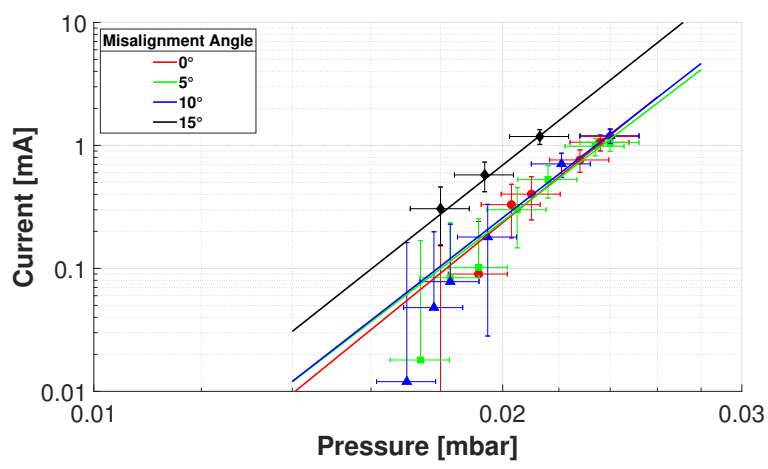

FIG. 12. WISP probe head at $1.5 \mathrm{kV}$ and relative misalignment of $0^{\circ}, 5^{\circ}, 10^{\circ}$, and $15^{\circ}$ to magnetic field vector

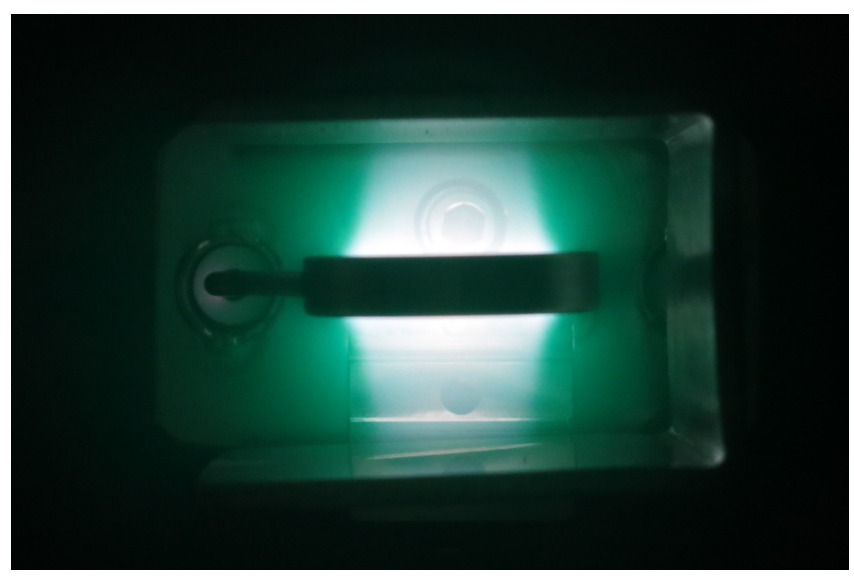

FIG. 13. Picture of a the plasma column inside the anode ring in He. The opening of the pinhole camera can be seen between the anode and the lower cathode plate

study what change in field direction is tolerable as well as to be able to define mounting tolerances, a systematic misalignment test was conducted at $0^{\circ}, 5^{\circ}, 10^{\circ}$, and $15^{\circ}$. Due to constraints with the vacuum system, these tests were done with a base pressure of $1 \times 10^{-3} \mathrm{mbar}$. Helium was used to sweep the pressure above that, but because of the residual water and air in the system, these measurements are only comparable among each other. Therefore, measurements were repeated with these conditions for the $0^{\circ}$ alignment. The four different angles were tested at all seven anode potentials described in subsection III B. Figure 12 shows the results at $1.5 \mathrm{kV}$ anode potential, showing the measured ion current during pressure curves for the four different angles. The behavior is exemplary for all other tested anode potentials. The curves of $0^{\circ}$, $5^{\circ}$, and $10^{\circ}$ lay on top of each other, while the $15^{\circ}$ curve shows a higher ion current. A small change in alignment of up to \pm $10^{\circ}$ doesn't seem to have an effect on the ion current measurement while the current at $15^{\circ}$ increases by a factor of about 2.4. When changing the magnetic field direction relative to the probe head, the plasma column (Fig. 13) inside the ring anode follows the tilt of the field. At $15^{\circ}$ the plasma column is tilted so far that it touches the anode and brings that spot to a glow. This leads to secondary electron emissions which is a likely reason for the increase in ion current. 


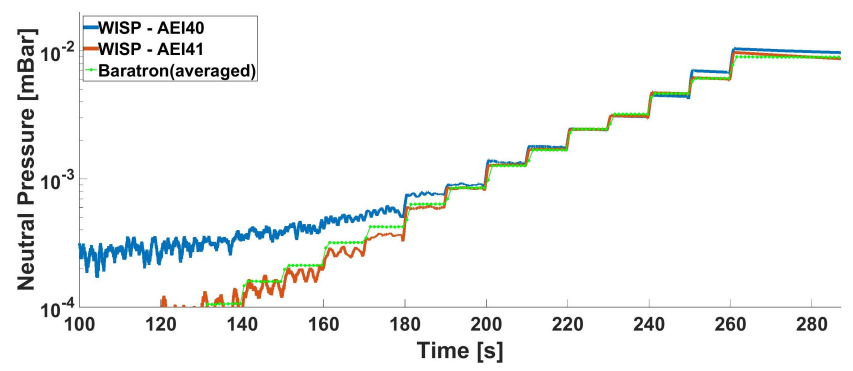

FIG. 14. The plot shows the pressure calibration curve that was conducted at the end of the campaign (ID: 20181010.001). The commercial baratrons serve as a reference that the other neutral gas gauges are calibrated against. In this plot the WISP signal is calibrated to the same calibration curve that was conducted at the beginning of the campaign (ID: 20180703.507). The calibration is still valid.

\section{F. Aging}

Penning gauges are known to deteriorate over time, making a re-calibration or an exchange of the anode and cathode necessary. Since the WISP probe head itself is cheap and anode and cathode are welded in, it is more cost effective to change the entire probe head if necessary. When using a new probe head, the inside of the anode ring has some initial roughness to it that the plasma slowly polishes off. This was observed through the release of sparks in the first minutes of running a new probe head. This effect can be minimized by polishing the anode ring during manufacturing, however it is recommended to run the probe head for 5-10 minutes at a relatively high pressure before calibrating it. A non polished probe head will show a higher ion current than a polished one. If calibration is done before this pre aging process, it will quickly be invalid. To study the effect of long term operation on the probe head, multiple in-situ calibrations (Subsec. IV C) were performed at $\mathrm{W} 7-\mathrm{X}$ throughout the operation phase $1.2 \mathrm{~b}^{18}$. While the gauges were running throughout an entire runday, the pressure was only high enough to measure current during plasma discharges, but not during the intermittent times. Figure 14 shows the standard neutral gas calibration program at $\mathrm{W} 7-\mathrm{X}$, in this case from the end of the campaign. The calibration procedure will be discussed in more detail in subsection IV C. The WISP curves from port AEI40 and AEI41 are unfiltered with the calibration factor from the very first calibration of the campaign but still agree with the pressure measured by the baratrons. The WISP gauge still shows the correct pressures and therefore was not affected by a significant aging throughout the 127 minutes of measurements. The noise and the offset in AEI40 at lower pressures are due to the ammeters used and will be replaced by more sensitive ones for future campaigns.

\section{SUCCESSFUL IMPLEMENTATION AT W7-X}

At W7-X the WISP manometers were mounted at three positions in module 4. Due to the easier removal of the probe head for maintenance purposes, a plugin design, based on the design for the hot cathode ion gauges ${ }^{19}$, was chosen. One in the top divertor pump gap (AEI41), one in the bottom divertor pump gap (AEI40), and one on the midplane (AEE41), allow- ing a good poloidal coverage in the module.

\section{A. Setup of assembly}

The plugins were mounted on DN63CF flanges and can be split into three main components. The actual probe head space in the front, an immersion tube which holds the probe head in place and provides the infrastructure, and an interface section which also serves as a vacuum barrier. Everything on the vacuum side is covered by stainless steel components with gaps < $0.7 \mathrm{~mm}$ between parts to allow pumping but shield from electron cyclotron resonance heating (ECRH) stray radiation. To reduce the amount of noise in the signal of the current measurement, both the high voltage anode and the ground cathode return were completely insulated from the machine.

\section{Probe head space}

The probe head space is confined by a $100 \mathrm{~mm}$ by $\varnothing 58$ $\mathrm{mm}$ stainless steel cylinder. The front plate is designed as a hole diaphragm (Fig. 16) made up of $75 \varnothing 0.7 \mathrm{~mm}$ holes that block out ECRH but allow a gas exchange. The probe head is mounted on an adjustment unit to be aligned to the magnetic field of the standard configuration, as seen in Fig. 3. The maximum directional change of magnetic field between different configurations is $2.5^{\circ}$ and is therefor well within the misalignment tolerances as discussed in subsection III E. The adjustment unit was manufactured out of copper to allow for a better heat transfer from the probe head to the immersion tube.

\section{Immersion tube}

The immersion tube holds the probe head in its designated place and guides two copper wires and the fiber over $2.2 \mathrm{~m}$ from the feed-through, through the cryostat, to the probe head space. The copper wires are insulated by ceramic tubes, ceramic pearls, and custom boron nitride covers at the screw joints. The center tube is at atmospheric pressure and could later be upgraded for active water cooling. This tube is surrounded by fins for stability with slits that guide the fiber and wires as seen in Fig. 17

\section{Interface}

The DN63CF flange serves as the vacuum barrier and has two electrical feed-throughs, one for the anode potential and one for the cathode return, which are both rated for $5 \mathrm{kV}$. A DN16CF flange was added for the SMA fiber feed-through, as shown in figure 18. On the air side the two electrical feedthroughs are connected to an SHV connector.

\section{B. Spectroscopic set-up at W7-X}

On the air side a $1 \mathrm{~mm}$ fiber is attached to the DN16CF fiber feedthrough, which guides the light of the WISP discharge 


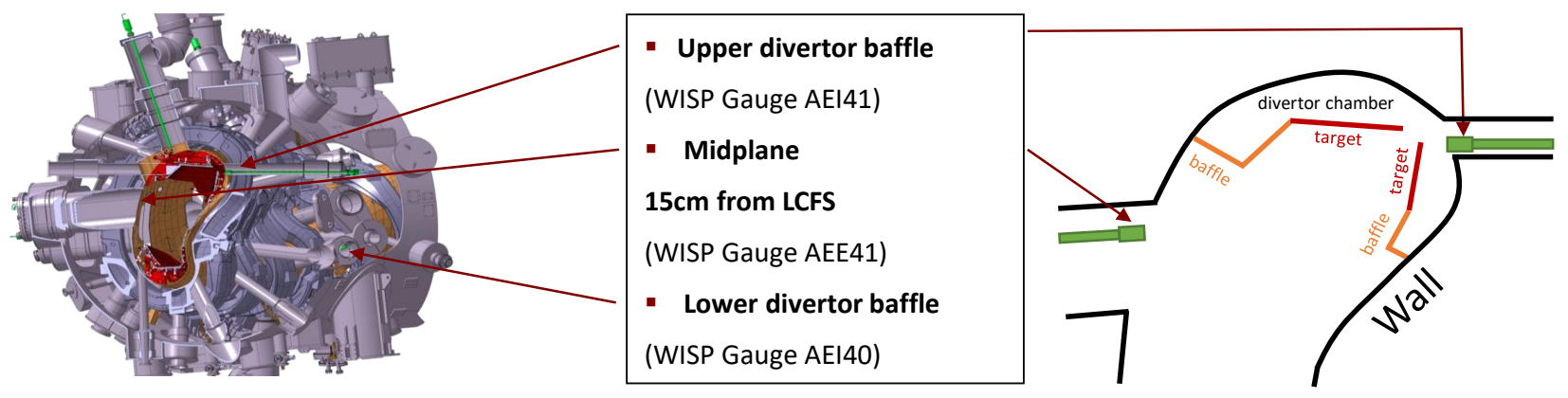

FIG. 15. (a) Cross section of module 4 of W7-X with the three mounting positions of the WISP gauge. (b) Cross section as a sketch with the plasma facing components and the relative positions of the WISP gauges.

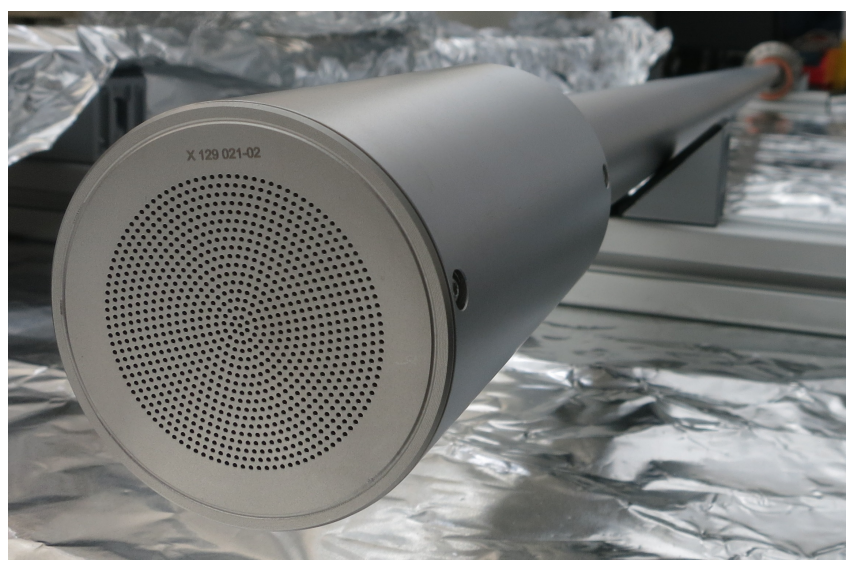

FIG. 16. Picture of a the hole diaphragm on the front plate of the probe head space

into a Filterscope $\mathrm{e}^{20}$ in the spectroscopy laboratory. The incoming beam is split into four separate beams for the four Filterscope channels each gauge uses. Each channel is equipped with a narrow band pass filter $( \pm 1 \mathrm{~nm})$ so that the photomultiplier tubes will only detect the desired wavelengths. For $\mathrm{H}$ measurements an $H_{\alpha}$ filter and for $\mathrm{He}$ a $667.8 \mathrm{~nm}$, a 706.5 $\mathrm{nm}$, and a $728.1 \mathrm{~nm}$ filter were used. Therefore, each channel monitors one single spectral line and measures its intensity. While this approach allows for large light throughput, it can suffer from interference from molecular features and background at $\mathrm{He}$ concentrations below $1 \%$. At such low concentrations the neutral $\mathrm{He}$ emission line gets weaker and the molecular spectra of hydrogen isotopes can dominate the emission within the spectral band around the He-I line. Instead of using filtered photodetectors, another study utilized dispersive spectroscopy to avoid such interference and achieved measurements down to the $0.1 \%$ level $^{21}$.

\section{Calibration at W7-X}

The calibration of the WISP gauges for OP1.2b was conducted in-situ, due to the magnetic field dependence of the measurement principle. This allowed to correct for potential misalignment along the magnetic field vector. It was conducted multiple times for 100\% Hydrogen and 100\% Helium, once at the beginning and once at the end of the campaign. Additionally, partial pressure calibrations were conducted in

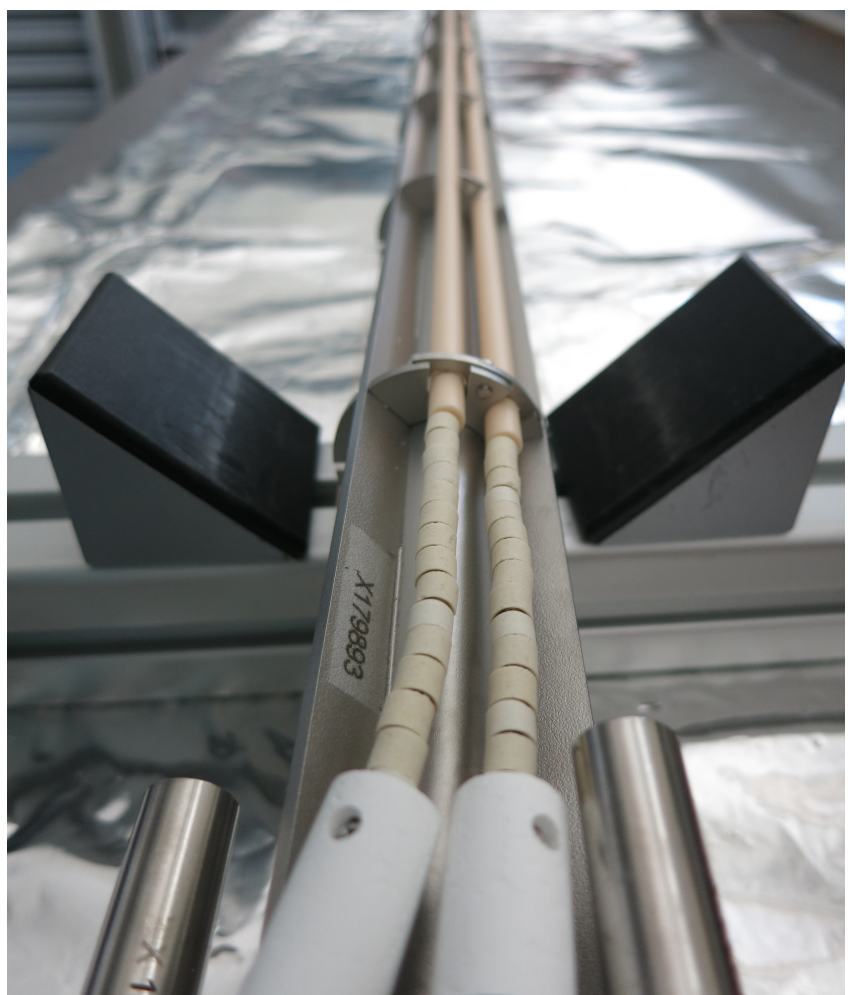

FIG. 17. Picture of the inside of the immersion tube without the surrounding ECRH cover. The fins as well as the insulated copper wires can be seen from the feed-through looking towards the probe head space.

$5 \%, 10 \%, 20 \%$, and 50\% Helium in Hydrogen during the first calibration. The calibration was conducted as increasing steps of constant pressure, starting at background pressure of about $1 \times 10^{-7}$ mbar up to $9 \times 10^{-3}$ mbar in 21 logarithmic equidistant steps. Of these 21 steps, not all were realized due to insufficient response of the gas valves, however 17 steps starting at $1 \times 10^{-6}$ mbar could reliably be realized. Constant pressure was achieved by closing all gate valves from the plasma vessel to the vacuum pumps and puffing defined amounts of gas into the plasma vessel. Each step had a flat top duration of $10 \mathrm{~s}$ with an additional $1 \mathrm{~s}$ for equilibration of gas throughout the torus system after the asymmetrical puff. The receded vacuum monitoring systems, consisting of a Penning and a Pirani gauge and partially augmented with two capacitance manometers, were used as pressure reference. Especially the 


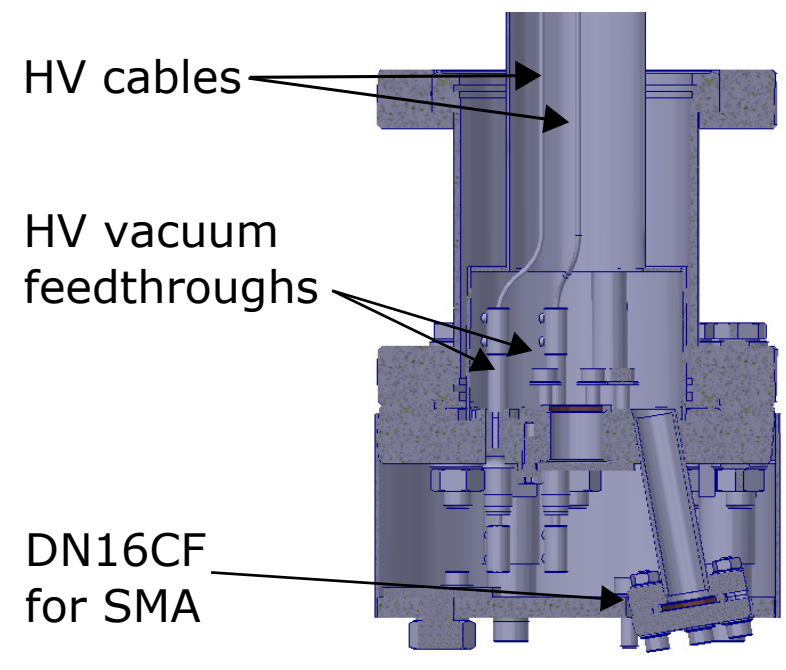

FIG. 18. Cross-section of the interface section with the SHV and SMA connectors

capacitance manometers provided a high-reliable, gas species independent, total pressure reference. These were mounted throughout the machine and were averaged to create the stair curve seen in Fig. 14 that was used as a reference to calibrate against. The WISP manometers were calibrated from $5 \times 10^{-5}$ mbar to $9 \times 10^{-3}$ mbar, however the upper limit was due to limitations in the vacuum system. The calibration function was extrapolated beyond the calibration and measurements of up to $1 \times 10^{-1}$ mbar were achieved. Also the deviations from the power law scaling at low pressures, as discussed in section III, could not be found at W7-X.

\section{Comparison with ASDEX pressure gauges}

At W7-X the WISP gauge was operated in the $2.5 \mathrm{~T}$ field at $2 \mathrm{kV}$ anode potential and would read pressures reliably between $1 \times 10^{-1}$ mbar and $1 \times 10^{-4}$ mbar at a time resolution of up to $1 \mathrm{kHz}$ at higher pressures. While the time resolution of the ion current measurement is not affected by the pressure, the time resolution of the line intensity measurement potentially needs adjustment for longer integration times as the line intensities get weaker with a decrease in pressure. All gauges worked very reliably throughout OP1.2b without failures. The ASDEX-type pressure gauges (APG) at $\mathrm{W} 7-\mathrm{X}^{19}$ can measure at a lower pressure, starting at $5 \times 10^{-7}$ mbar up to $4 \times 10^{-2}$ mbar at a time resolution of up to $5 \mathrm{kHz}$. Both gauges have an accuracy of about $15 \%$. The APG's are suitable for a wider pressure range and can provide a faster time resolution, specially at lower pressures. While the APG's with tungsten filament weren't as reliable due to jxB failures, the issue was resolved with the $\mathrm{LaB}_{6}$ electron emitters in the now called crystal pressure gauge ${ }^{22}$ (CPG). The WISP gauges have their strength in higher pressures, which occur inside the divertor. Besides being capable of measuring higher pressures than the CPG, the partial pressures can be extracted from spectral line ratios measurements with the WISP gauge, which is crucial for exhaust studies.

The ASDEX pressure gauges were used for comparison with the WISP gauges as both systems were operated at W7-

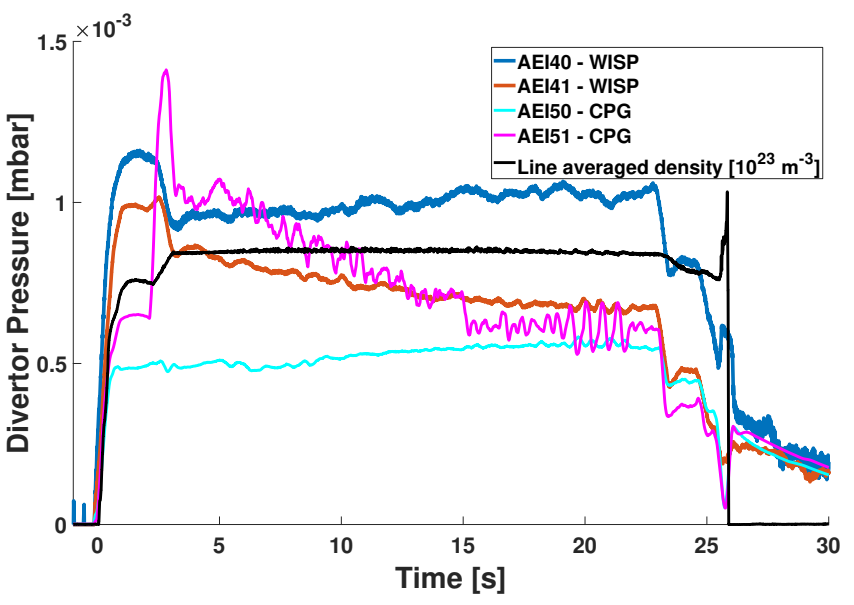

FIG. 19. Comparison of divertor pump gap pressure measurements in module 4 (WISP) and module 5 (CPG) of program ID 20181016.018 shows a general agreement between WISP and CPG

$\mathrm{X}$. However, there are multiple other in-situ pressure gauges that have been developed to be used on large fusion devices. Notably the "In-vessel Neutral Pressure", INP gauge, and the "Princeton Divertor eXperiment", PDX, type Penning gauge that were operated between $0.45 \mathrm{~T}$ and $0.6 \mathrm{~T}$ at $\mathrm{NSTX}^{23}$ as well as the miniature Penning gauge at Alcator C-Mod capable of millisecond time resolution at ambient magnetic fields in the range of 2 to $8 \mathrm{~T}^{24,25}$. Figure 19 shows pressure measurements and line integrated density of a discharge. In the first three seconds the plasma starts up with a density ramp up, after which the density is kept constant until the last few seconds of the discharge before it spikes at the shut off. The pressure measurements are from a set of two WISP gauges in module four and two CPG's in the same ports in module five. Both diagnostics scale comparably and show similar effects. WISP in AEI41 and CPG in AEI51, which are both mounted in the top divertor pump gap show a constant decay of the divertor pressure with a four peak ripple at second 20, while the two gauges in the lower divertor AEI40 and AEI50 show a rather constant divertor pressure. Differences in pressure can be explained through local effects in the different modules based on asymmetries or like the strong peak in AEI51 at $3 \mathrm{~s}$, due to localized gas puffing close to the gauge. When considering these effects, it can be concluded that measurements from both diagnostics are comparable.

While the time resolution of the WISP gauges is slower than of the ASDEX-type gauges, it is fast enough to see the effect of individual pellets on the neutral pressure at $10 \mathrm{~Hz}$. This effect is shown in figure 20 where the line averaged density is shown on the right-hand side and the $\mathrm{H}$ partial pressure measured by the WISP gauge in port AEI40 on the left-hand side. The density shows the plasma start up during the first second and a steep spiked density increase starting at $3 \mathrm{~s}$. Each spike is an individual pellet entering the plasma and a density decay afterwards until the next pellet enters the plasma. These spikes that are formed by individual pellets can also be seen in the partial pressure measurement of the WISP gauge. While the exact time response of the WISP gauge is still under investigation, this shows that effects from pellets entering at 10 Hz can be studied.

He partial pressures were measured during He gas injec- 


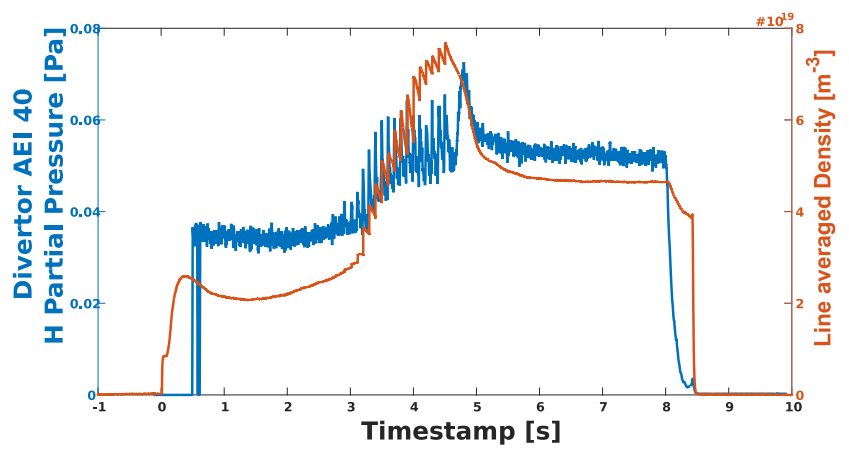

FIG. 20. Hydrogen partial pressure and line averaged density over time of program ID 20181010.025 with $10 \mathrm{~Hz}$ pellets

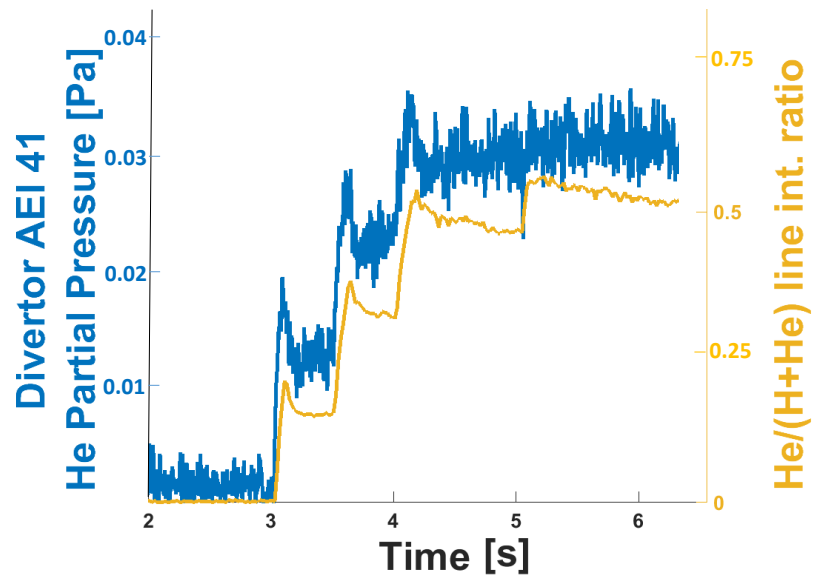

FIG. 21. He partial pressure on the left and He concentration measured by charge exchange spectroscopy on the right over time of program ID 20181010.020 with three He gas injections at $3 \mathrm{~s}, 3.5 \mathrm{~s}$, and $4 \mathrm{~s}$.

tion experiments into a $\mathrm{H}$ plasma. The He partial pressure measured by the WISP gauge in port AEI40 is shown in blue on the left hand-side and the $\mathrm{He} /(\mathrm{H}+\mathrm{He})$ ratio from charge exchange spectroscopy in yellow on the right-hand side of figure 21. The three He gas injections at $3 \mathrm{~s}, 3.5 \mathrm{~s}$, and $4 \mathrm{~s}$ can be seen in both signals. Each injection is followed by a sudden jump in He partial pressure, followed by a fast decay until a plateau or very slow decay is reached. The gas injection increases the neutral He household, seen in the sudden increase of partial pressure. The He reservoir is then depleted on a fast time scale by ionization, as more and more neutral He particles enter the plasma, until an equilibrium is reached about $200 \mathrm{~ms}$ after the injection. This demonstrates the He partial pressure measurement capabilities in a $\mathrm{H}$ background plasma at multiple $\mathrm{He}$ to $\mathrm{H}$ concentrations.

\section{SUMMARY}

The Wisconsin In-Situ Penning (WISP) probe head was developed and optimized for partial pressure measurements in the divertor pressure regime of fusion devices. Operation between $0.07 \mathrm{~T}$ and $3.5 \mathrm{~T}$ and with anode potentials between $1.25 \mathrm{kV}$ and $5 \mathrm{kV}$ has been shown. The pressure range is de- pendant on the anode potential and the magnetic field. The magnetic field is usually dictated by the device and can therefore be a limiting factor, especially at higher fields since the lower end of the operating regime shrinks with an increase in field. The anode potential can be used as a tool to somewhat mitigate this effect by moving the operational range from left to right. The highest achievable pressure was $0.1 \mathrm{mbar}$ which was limited by the maximum acceptable pressure of the vacuum pumping system at MDPX. As long as the WISP probe head can withstand the deposited input power no reason could be seen why higher pressures should not be achievable. A misalignment of $\pm 10^{\circ}$ has no effect on the measurement. While the ion current measurement is gas dependent, this can be avoided by the light intensity measurements for partial pressures. It was shown that there is negligible cross talk between $\mathrm{He}$ and $\mathrm{H}$ measurements, and the line intensity is proportional to the partial pressure. The ion current as well as the light intensity curves can be described by power law functions. At MDPX there was a deviation of the power law fit at low pressures, which could not be found at other devices. Therefor the presented data serves as a visualization of the behavior and can not serve as calibration functions. An in-situ calibration is inevitable. The error and sensitivity of the ion current measurement at low currents could be increased by more sensitive ammeters. The WISP head was shown to be a robust component, insensitive to venting of the vacuum environment. The WISP probe can be vented without permanent damage or requiring a new calibration after venting. The diagnostic was successfully implemented at W7-X on a plugin design and measured reliably throughout the entire OP1.2b campaign. The gauges were in-situ calibrated and showed general agreement with other neutral gas diagnostics. The calibration functioned followed a power law fit throughout the entire operating range without any deviations. Future work will be the inclusion of a collisional radiative model to calculate density and temperature of the Penning discharge to analytically derive a partial pressure as well as a more extensive exploration of other light impurities.

\section{ACKNOWLEDGMENTS}

This work was funded in part by the Department of Energy under grant DE-SC0014210. The author would like to give a special thanks to Edward Thomas Jr, Darrick Artis and the rest of the MDPX team at Auburn University for granting access to MDPX as well as their continuous help, support, and time. The operation of the MDPX device is funded by the Department of Energy under Award Number DE-SC0019176, the NSF-DOE Partnership Program in Basic Plasma Science and Engineering (PHY-1613087 / DE - SC0016330), and the NSF-EPSCoR program (OIA-1655280). The MDPX device was originally designed and built with funding from the NSFMRI program (NSF-1126067).

This work has been carried out within the framework of the EUROfusion Consortium and has received funding from the Euratom research and training programme 2014-2018 and 2019-2020 under grant agreement No 633053. The views and opinions expressed herein do not necessarily reflect those of the European Commission.

\footnotetext{
${ }^{1}$ D. Reiter, G. Wolf, and H. Kever, Nuclear Fusion 30, 2141 (1990).
} 
${ }^{2}$ K. Ida, M. Yoshinuma, B. Wieland, M. Goto, Y. Nakamura, M. Kobayashi, I. Murakami, and C. Moon, Review of Scientific Instruments 86, 123514 (2015).

${ }^{3}$ A. Kappatou, R. McDermott, T. Pütterich, R. Dux, B. Geiger, R. Jaspers, A. Donné, E. Viezzer, M. Cavedon, et al., Plasma Physics and Controlled Fusion 60, 055006 (2018).

${ }^{4}$ H.-S. Bosch, G. Haas, and M. Lörcher, Journal of nuclear materials 196, 1074 (1992).

${ }^{5}$ K. Finken, K. Dippel, W. Baek, and A. Hardtke, Review of scientific instruments 63, 1 (1992).

${ }^{6}$ F. M. Penning, physica 3, 873 (1936).

${ }^{7}$ T. Denner, K. Finken, and G. Mank, Review of scientific instruments 67, 3515 (1996)

${ }^{8}$ M. Wade, D. Hillis, J. Hogan, M. Mahdavi, R. Maingi, W. West, N. Brooks, K. Burrell, R. Groebner, G. Jackson, et al., Physical review letters 74, 2702 (1995).

${ }^{9}$ D. Hillis, C. Klepper, M. Von Hellermann, J. Ehrenberg, K. Finken, and G. Mank, Fusion engineering and design 34, 347 (1997).

${ }^{10}$ C. C. Klepper, T. Biewer, V. B. Graves, P. Andrew, P. Lukens, C. Marcus, M. Shimada, S. Hughes, B. Boussier, D. Johnson, et al., Fusion Engineering and Design 96, 803 (2015).

${ }^{11}$ K. Flesch, T. Kremeyer, O. Schmitz, V. Soukhanovskii, and U. Wenzel, Review of Scientific Instruments 87, 11E529 (2016).

${ }^{12}$ E. Thomas, U. Konopka, D. Artis, B. Lynch, S. Leblanc, S. Adams, R. Merlino, and M. Rosenberg, Journal of Plasma Physics 81 (2015).

${ }^{13}$ H. Saitoh, T. S. Pedersen, U. Hergenhahn, et al., Journal of Physics: Conference Series 505, 012045 (2014).
${ }^{14}$ O. Schmitz, I. Beigman, L. Vainshtein, B. Schweer, M. Kantor, A. Pospieszczyk, Y. Xu, M. Krychowiak, M. Lehnen, U. Samm, et al., Plasma physics and controlled fusion 50, 115004 (2008).

${ }^{15}$ G. Pacher, H. Pacher, G. Janeschitz, A. Kukushkin, V. Kotov, and D. Reiter, Nuclear fusion 47, 469 (2007).

${ }^{16}$ D. Douai, T. Goodman, A. Isayama, et al., Nuclear Fusion 58, 026018 (2017).

${ }^{17}$ C. Klepper, D. Hillis, M. Wade, et al., Review of scientific instruments 68 , 400 (1997).

${ }^{18}$ H.-S. Bosch, R. Brakel, T. Braeuer, V. Bykov, P. van Eeten, J.-H. Feist, F. Füllenbach, M. Gasparotto, H. Grote, T. Klinger, et al., Nuclear Fusion 57, 116015 (2017).

${ }^{19}$ U. Wenzel, T. Kremeyer, G. Schlisio, M. Marquardt, T. Pedersen, O. Schmitz, B. Mackie, J. Maisano-Brown, et al., Journal of Instrumentation 12, C09008 (2017)

${ }^{20}$ R. Colchin, D. Hillis, R. Maingi, C. Klepper, and N. Brooks, Review of scientific instruments 74, 2068 (2003).

${ }^{21}$ C. Klepper, T. Biewer, C. Marcus, et al., Journal of Instrumentation 12, C10012 (2017).

${ }^{22}$ U. Wenzel, T. Pedersen, M. Marquardt, and M. Singer, Review of Scientific Instruments 89, 033503 (2018).

${ }^{23}$ R. Raman, H. Kugel, T. Provost, R. Gernhardt, T. Jarboe, and M. Bell, Review of scientific instruments 74, 1900 (2003).

${ }^{24}$ B. Lipschultz, B. LaBombard, C. Pitcher, et al., Plasma physics and controlled fusion 44, 733 (2002).

${ }^{25}$ B. LaBombard, PSFC/RR-15-12 (2015). 